\title{
Stable Conical Regularization by Constructible Dilating Cones with an Application to $L^{p}$-constrained Optimization Problems
}

\author{
Baasansuren Jadamba, Akhtar A. Khan and Miguel Sama*
}

\begin{abstract}
We study a convex constrained optimization problem that suffers from the lack of Slater-type constraint qualification. By employing a constructible representation of the constraint cone, we devise a new family of dilating cones and use it to introduce a family of regularized problems. We establish novel stability estimates for the regularized problems in terms of the regularization parameter. To show the feasibility and efficiency of the proposed framework, we present applications to some $L^{p}$-constrained least-squares problems.
\end{abstract}

\section{Introduction}

Let $U$ and $V$ be real Hilbert spaces, let $Y$ be a real Banach space, and let $\|\cdot\|_{U},\|\cdot\|_{V}$, and $\|\cdot\|_{Y}$ be their norms. Let $K \subset Y$ be a closed, convex, and pointed cone that induces a partial ordering $\leq_{K}$ on $Y$. Denoting by $Y^{*}$ the dual space of $Y$, by $K^{*}=\left\{y^{*} \in Y^{*} \mid\right.$ $y^{*}(k) \geq 0$ for every $\left.k \in K\right\}$, we represent the positive dual of $K$.

We focus on the following convex minimization problem:

$$
\text { Minimize } J(u):=\frac{1}{2}\left\|S u-z_{d}\right\|_{V}^{2}+\frac{\kappa}{2}\left\|u-u_{d}\right\|_{U}^{2} \text { subject to } G u \leq_{K} w, u \in U .
$$

Here $S: U \rightarrow V, G: U \rightarrow Y$ are linear bounded operators, $\kappa>0$ is a given parameter, and $u_{d} \in U, z_{d} \in V$, and $w \in Y$ are given elements. Clearly, problem (1.1) has a unique solution $\bar{u}$. However, there are important examples where the solution cannot be computed using the Karush-Kuhn-Tucker (KKT) conditions. One remedy then is to associate (1.1) to a family of regularized optimization problems which can be solved through the KKT conditions provided that the regularized solutions enjoy nice properties. There are multiple ways to construct a family of regularized problems. For example, the conical regularization, proposed in [17, employs a family of dilating cones of $K$ to construct the regularized family (see $[5,10,22]$ ). Recently, this idea has been applied to PDE constrained models (see $15,17,19,20]$ ). For $\varepsilon \in(0,1)$, we denote by $\left\{\mathcal{K}_{\varepsilon}\right\} \subset Y$, a

Received January 10, 2018; Accepted November 1, 2018.

Communicated by Jein-Shan Chen.

2010 Mathematics Subject Classification. 90C20, 90C31, 90C46.

Key words and phrases. perturbation theory, convex optimization, half-space representation, conical regularization, dilating cones.

*Corresponding author. 
family of dilating cones associated with $K$. That is, $\left\{\mathcal{K}_{\varepsilon}\right\}$ is a family of closed, convex, and pointed cones with nonempty interior such that, firstly, $K \backslash\{0\} \subset \operatorname{int}\left(\mathcal{K}_{\varepsilon}\right)$, for every $\varepsilon \in(0,1)$, and secondly, $K=\bigcap_{\varepsilon>0} \mathcal{K}_{\varepsilon}$.

The regularized family is then obtained by replacing $K$ in 1.1 by $\mathcal{K}_{\varepsilon}$ as follows:

$$
\text { Minimize } J(u):=\frac{1}{2}\left\|S u-z_{d}\right\|_{V}^{2}+\frac{\kappa}{2}\left\|u-u_{d}\right\|_{U}^{2} \text { subject to } G u \leq_{\mathcal{K}_{\varepsilon}} w, u \in U .
$$

It was shown in [17] that the regularized solutions $u_{\varepsilon}$ converge to $\bar{u}$ and under an additional mild condition, see (3.3), the regularized solution $u_{\varepsilon}$ can be computed using the KKT system: There is a multiplier $\mu_{\varepsilon}^{*} \in \mathcal{K}_{\varepsilon}^{*}$ such that

$$
\begin{aligned}
D J\left(u_{\varepsilon}\right)+\mu_{\varepsilon}^{*} \circ G & =0, \\
\mu_{\varepsilon}^{*}\left(G u_{\varepsilon}-w\right) & =0, \\
G u_{\varepsilon}-w & \leq \mathcal{K}_{\varepsilon} 0,
\end{aligned}
$$

where $\circ$ denotes the usual composition operator.

We note that 17] developed the conical regularization scheme assuming that the constraint space $Y$ is a real Hilbert space. However, the convergence results [17, Theorems 2.1, 3.2], and the KKT conditions [17, Theorem 3.3] remain valid for the case when the constraint space $Y$ is a real Banach space. This is because the convergence results mainly rely on the continuity of the operator $G$ and the closedness and convexity of the cone $K$, whereas the KKT conditions follow from the fact that the dilating cones $\mathcal{K}_{\varepsilon}$ are solid.

In this work, we construct a new family of dilating cones which is similar to the Henig dilating cones proposed by Henig [10] in a finite-dimensional setting and then extended by Borwein and Zhuang [5] to general spaces. We construct this new family using a halfspace representation of the cone $K$ (see $[4,14,16]$ ) and show that it enjoys the same set of nice properties as the Henig dilating cones but is more tractable from a computational stand point. Using this constructible family of dilating cones, we propose a new regularization scheme. We estimate the regularization error for (1.1) for the new regularization scheme and for the Henig regularization scheme studied in [17]. More precisely, for the two cases, we establish an upper bound of the regularization error for the optimal values of (1.1) and $(1.2)$, the norms of the regularized multipliers and the regularization parameter (see Theorems 3.2 and 3.6. Apart from its theoretical interest, this bound is particularly useful for problems where a bound on the optimal value of the original problem is available. Furthermore, for the Henig regularization, we establish an a priori regularization error estimate of order $\mathcal{O}(\sqrt{\varepsilon})$ when the problem is regular (see Theorem 3.3). The same result is given for the new constructible regularization scheme (Theorem 3.8), assuming that a Slater condition is verified. For this, we employ an equivalent $\ell^{\infty}$ reformulation of the abstract optimization problem (1.1) and apply a known stability result of Alt [1. As a 
consequence, in both cases, when $Y$ is in addition a reflexive Banach space, we characterize the regular problems as those which have norm bounded associated regularized multipliers. We note that these estimates are general and hold for every cone $K$ in the Banach space $Y$.

The computable form of the new family of dilating cones allows us to construct a family of approximate problems in a natural way. We apply this scheme to an important case when the constraint space is an $L^{p}$ function space with $1 \leq p \leq \infty$. For this case, we provide an explicit construction of the dilating cones for each exponent $p \in[1, \infty]$. We conduct a numerical experiment for a known example of nonregular problem with $L^{p}$ constraints and perform a systematic study of the new constructible regularization and give a full comparison with the known Henig regularization.

Finally, we describe the notions adopted in this paper. For a Banach space $X$, we denote its dual by $X^{*}$ and its norm by $\|\cdot\|_{X}$. The inner product of a Hilbert space $H$ will be denoted by $\langle\cdot, \cdot\rangle_{H}$. For each $x^{*} \in X^{*}$, by $H_{x^{*}}=\left\{x \in X: x^{*}(x) \geq 0\right\}$, we denote its associated halfspace. By $\left(\ell^{\infty},\|\cdot\|_{\ell^{\infty}}\right)$, we denote the space of bounded sequences $v=\left\{v_{i}\right\}_{i \in \mathbb{N}} \subset \mathbb{R}$ with norm $\|v\|_{\ell^{\infty}}=\max \left\{\left|v_{i}\right|: i \in \mathbb{N}\right\}$. The cone of positive coordinates $\ell_{+}^{\infty}$ is given by $\ell_{+}^{\infty}=\left\{v=\left\{v_{i}\right\}_{i \in \mathbb{N}} \in \ell^{\infty}: v_{i} \geq 0\right.$ for every $\left.i \in \mathbb{N}\right\}$. The space of all bounded linear operators from $U$ to $Y$ is denoted by $L(U, Y)$. For a linear operator $S: U \rightarrow Y$, by $S^{*}: Y^{*} \rightarrow U^{*}$ we denote the adjoint operator. For Hilbert spaces, we will follow the usual convention of defining $S^{*}: Y \rightarrow U$ by $\langle S u, y\rangle_{Y}=\left\langle u, S^{*} y\right\rangle_{U}$ for every $u \in U, y \in Y$. For $J: U \rightarrow Y$, by $D J(\bar{u})$ and $D^{2} J(\bar{u})$, we denote the first-order and the second-order Fréchet derivative at a point $\bar{u} \in U$. By $c>0$, we will denote a generic positive constant.

\section{A constructible family of dilating cones}

Recall that $Y$ is a Banach space and $K \subset Y$ is a closed, convex, and pointed cone. We aim to construct a family of dilating cones $\left\{\mathcal{K}_{\varepsilon}\right\}$ of $K$, where $\varepsilon \in(0,1)$. This construction is based on a halfspace decoupling of the cone $K$ (see [14]) and the notion of the Henig dilating cone introduced by Henig in finite-dimensional setting [10] and later extended to general spaces by Borwein and Zhuang [5]. We assume that the cone $K$ has a closed and convex base $\Theta \subset K$ such that $0 \notin \Theta$ and

$$
K=\bigcup_{\lambda \geq 0}\{\lambda \theta: \theta \in \Theta\}
$$

Without loss of generality (see for [18, Theorem 2.2.12]), we assume that the base $\Theta$ is given by a strictly positive functional $\beta^{*} \in K^{\natural}:=\left\{y^{*} \in Y^{*}: y^{*}(k)>0, \forall k \in K \backslash\{0\}\right\}$, that is, $\Theta=\left\{y \in K: \beta^{*}(y)=1\right\}$, where we normalize to $\left\|\beta^{*}\right\|_{Y^{*}}=1$. Given $\varepsilon>0$, the 
Henig dilating cone is then given by

$$
K_{\varepsilon}:=\operatorname{cl}\left[\operatorname{cone}\left(\Theta+\varepsilon B_{Y}\right)\right] \text {, }
$$

where $B_{Y}=\left\{v \in Y:\|v\|_{Y} \leq 1\right\}$ is the closed unit ball. It is known (see [5, Theorem 1.1]) that the Henig cone is a solid, closed, convex, and pointed cone such that $K \backslash\{0\} \subset \operatorname{int}\left(K_{\varepsilon}\right)$, for every $\varepsilon \in(0,1)$, and $K=\bigcap_{0<\varepsilon<1} K_{\varepsilon}$.

Before any advancement, we provide the following characterization of the dual cone $K_{\varepsilon}^{*}$ which is based on [17, Theorem 4.1]. We emphasize that although this characterization has initially been given for a Hilbert space, the proof only used the properties of normed spaces and hence it remains valid for a general Banach space.

Theorem 2.1. Let $\varepsilon \in(0,1)$. The dual of the Henig dilating cone $K_{\varepsilon}^{*}$ is given by

$$
K_{\varepsilon}^{*}=\{0\} \cup\left\{0 \neq \lambda^{*} \in K^{*}: \lambda^{*}(\theta) \geq \varepsilon\left\|\lambda^{*}\right\| \text { for every } \theta \in \Theta\right\} .
$$

Given $y^{*} \in Y^{*}$, by $y_{\varepsilon}^{*} \in Y^{*}$ we denote the functional

$$
y_{\varepsilon}^{*}:=\varepsilon \beta^{*}+(1-\varepsilon) y^{*},
$$

which, for sufficiently small $\varepsilon$, belongs to $K_{\varepsilon}^{*}$ as shown in the following:

Lemma 2.2. Let $y^{*} \in K^{*}$. If $\varepsilon \in(0,1)$ and $\left\|y^{*}\right\|_{Y^{*}} \leq 1$, then $y_{\varepsilon}^{*} \in K_{\varepsilon}^{*}$ with $\left\|y_{\varepsilon}^{*}\right\|_{Y^{*}} \leq 1$.

Proof. Clearly, for every $\theta \in \Theta$, we have

$$
y_{\varepsilon}^{*}(\theta)=\varepsilon \beta^{*}(\theta)+(1-\varepsilon) y^{*}(\theta)=\varepsilon+(1-\varepsilon) y^{*}(\theta) \geq \varepsilon .
$$

On the other hand, $\left\|y_{\varepsilon}^{*}\right\|_{Y^{*}} \leq \varepsilon\left\|\beta^{*}\right\|_{Y^{*}}+(1-\varepsilon)\left\|y^{*}\right\|_{Y^{*}} \leq \varepsilon+(1-\varepsilon)=1$, which implies that $\left\|y_{\varepsilon}^{*}\right\|_{Y^{*}} \leq 1$. By using $(2.2)$, we have

$$
\frac{y_{\varepsilon}^{*}(\theta)}{\left\|y_{\varepsilon}^{*}\right\|} \geq y_{\varepsilon}^{*}(\theta) \geq \varepsilon,
$$

which in view of Theorem 2.1 gives $y_{\varepsilon}^{*} \in K_{\varepsilon}^{*}$.

We recall that $K=\bigcap_{y^{*} \in K^{*}} H_{y^{*}}$ where $H_{y^{*}}$ is the positive half-space (see for example [25]). This allows us to give the following definition.

Definition 2.3. Let $I$ be an arbitrary nonempty index set and let $\left\{\lambda_{i}^{*}\right\}_{i \in I} \subset K^{*}$. The collection $\left\{H_{\lambda_{i}^{*}}\right\}_{i \in I}$ is said to be a representation of $K$ if $K=\bigcap_{i \in I} H_{\lambda_{i}^{*}}$.

In the following, $\left\{H_{\lambda_{i}^{*}}\right\}_{i \in I}$ is a fixed representation of $K$, where without loss of generality, $\left\{\lambda_{i}^{*}\right\}_{i \in I}$ are normalized so that $\left\|\lambda_{i}^{*}\right\|_{Y^{*}}=1$, for every $i \in I$. Following (2.1) and the given representation $\left\{H_{\lambda_{i}^{*}}\right\}_{i \in I}$ of $K$, for each $\lambda_{i}^{*} \in K^{*}$, we define, for any $i \in I$,

$$
\lambda_{i, \varepsilon}^{*}=\varepsilon \beta^{*}+(1-\varepsilon) \lambda_{i}^{*}
$$


For any $i \in I$ and $0<\varepsilon<1$, Lemma 2.2 yields $\left\|\lambda_{i, \varepsilon}^{*}\right\|_{Y^{*}} \leq 1$. For $0<\varepsilon<1$, we define

$$
C_{\varepsilon}:=\bigcap_{i \in I} H_{\lambda_{i, \varepsilon}^{*}}
$$

The following result shows that for $\varepsilon \in(0,1),\left\{C_{\varepsilon}\right\}$ is indeed a family of dilating cones.

Theorem 2.4. For $\varepsilon \in(0,1), C_{\varepsilon}$ is a solid, closed, convex, and pointed cone such that

(i) $K_{\varepsilon} \subset C_{\varepsilon}$.

(ii) $K \backslash\{0\} \subset \operatorname{int}\left(C_{\varepsilon}\right)$.

(iii) $K=\bigcap_{\varepsilon>0} C_{\varepsilon}$.

Proof. By definition, $C_{\varepsilon}$ is a closed and convex cone. Furthermore $\beta^{*} \in C_{\varepsilon}^{\natural}$, thus $C_{\varepsilon}$ is based and consequently pointed (see $[7$, Example 1.1.2.]). On the contrary there would exist $0 \neq c_{\varepsilon} \in C_{\varepsilon}$ such that

$$
\beta^{*}\left(c_{\varepsilon}\right) \leq 0
$$

By definition,

$$
\lambda_{i, \varepsilon}^{*}\left(c_{\varepsilon}\right)=(1-\varepsilon) \lambda_{i}^{*}\left(c_{\varepsilon}\right)+\varepsilon \beta^{*}\left(c_{\varepsilon}\right) \geq 0 \quad \Longrightarrow \quad \lambda_{i}^{*}\left(c_{\varepsilon}\right) \geq \frac{\varepsilon}{1-\varepsilon}\left|\beta^{*}\left(c_{\varepsilon}\right)\right| \geq 0
$$

for every $i \in \mathbb{N}$. Thus $c_{\varepsilon} \in \bigcap_{\varepsilon>0} H_{\lambda_{i}^{*}}=K$, which contradicts (2.5).

Moreover, by Lemma 2.2, $\lambda_{i, \varepsilon}^{*} \in K_{\varepsilon}^{*}$ and hence $K_{\varepsilon} \subset H_{\lambda_{i, \varepsilon}^{*}}$, for every $i \in I$. Consequently, $K_{\varepsilon} \subset \bigcap_{\varepsilon>0} H_{\lambda_{i, \varepsilon}^{*}}=C_{\varepsilon}$. This, in view of $K=\bigcap_{0<\varepsilon<1} K_{\varepsilon}$, yields $K \backslash\{0\} \subset$ $\operatorname{int}\left(K_{\varepsilon}\right) \subset \operatorname{int}\left(C_{\varepsilon}\right)$. For $K=\bigcap_{\varepsilon>0} C_{\varepsilon}$, we note that $K \subset \bigcap_{\varepsilon>0} C_{\varepsilon}$. For the converse, let $w \in \bigcap_{\varepsilon>0} C_{\varepsilon}$ be arbitrary. Then,

$$
\lambda_{i}^{*}(w) \geq \frac{\varepsilon}{\varepsilon-1} \beta^{*}(w) \quad \text { for every } i \in I, \varepsilon>0 .
$$

Since it is true for any $\varepsilon \in(0,1)$, we get $\lambda_{i}^{*}(w) \geq 0$ for each $i \in I$, implying that $w \in K$.

We conclude this section by showing that $K_{\varepsilon} \subset C_{\varepsilon}$ is in general strict.

Example 2.5. We set $Y=\mathbb{R}^{2}$ equipped with its Euclidean norm and the ordering $K=$ $\mathbb{R}_{+} \times\{0\}$. By identifying elements of the dual space with vectors, we consider the strictly positive functional $\beta^{*} \equiv(1,0)$ and the representation $\left\{H_{\lambda_{i}^{*}}\right\}_{i \in\{1,2,3\}}$ given by vectors $\lambda_{1}^{*} \equiv$ $(0,1), \lambda_{2}^{*} \equiv(0,-1), \lambda_{3}^{*} \equiv(1,0)$. Then,

$$
\begin{aligned}
& K_{\varepsilon}=\left\{(x, y) \in \mathbb{R}^{2}: \varepsilon\left(1-\varepsilon^{2}\right)^{-1 / 2} x \geq y \geq-\varepsilon\left(1-\varepsilon^{2}\right)^{-1 / 2} x, x \geq 0\right\}, \\
& C_{\varepsilon}=\left\{(x, y) \in \mathbb{R}^{2}: \varepsilon(1-\varepsilon)^{-1} x \geq y \geq-\varepsilon(1-\varepsilon)^{-1} x, x \geq 0\right\},
\end{aligned}
$$

which confirms that $K_{\varepsilon} \subsetneq C_{\varepsilon}$ for $\varepsilon \in(0,1)$. 


\section{Stability estimates}

Regularized family 1.2 covers two cases of interest. For the choice $\left\{\mathcal{K}_{\varepsilon}\right\}=\left\{K_{\varepsilon}\right\}$, we obtain the Henig conical regularization embedded in

$$
\text { Minimize } J(u):=\frac{1}{2}\left\|S u-z_{d}\right\|_{V}^{2}+\frac{\kappa}{2}\left\|u-u_{d}\right\|_{U}^{2} \text { subject to } G u \leq_{K_{\varepsilon}} w, u \in U .
$$

Moreover, for $\left\{\mathcal{K}_{\varepsilon}\right\}=\left\{C_{\varepsilon}\right\}$, we get the constructible regularized problems

$$
\text { Minimize } J(u):=\frac{1}{2}\left\|S u-z_{d}\right\|_{V}^{2}+\frac{\kappa}{2}\left\|u-u_{d}\right\|_{U}^{2} \text { subject to } G u \leq C_{\varepsilon} w, u \in U .
$$

We now aim to establish stability estimates for the regularization error in both of the cases. We will denote by $u_{\varepsilon}, u_{\varepsilon}^{H}$, and $u_{\varepsilon}^{C}$, the solutions to (1.2), (3.1), and 3.2), respectively. We will use the notation $\bar{q}=G \bar{u}-w$, and $q_{\varepsilon}=G u_{\varepsilon}-w, q_{\varepsilon}^{H}=G u_{\varepsilon}^{H}-w$, and $q_{\varepsilon}^{C}=G u_{\varepsilon}^{C}-w$ for $\varepsilon>0$ sufficiently small. In the rest of the paper, we impose the following mild assumption

$$
\bar{q} \neq 0
$$

which essentially prevents problem (1.1) from being a pure equality constraint problem. Indeed, if $\bar{q}=G \bar{u}-w=0$, then $\bar{u}$ is also feasible, and hence a solution, of the simpler equality problem:

$$
\text { Minimize } \frac{1}{2}\left\|S u-z_{d}\right\|_{V}^{2}+\frac{\kappa}{2}\left\|u-u_{d}\right\|_{U}^{2} \text { subject to } G u=w, u \in U .
$$

Furthermore, (3.3) provides a nontrivial feasible point such that the regularized optimality system is solvable (see [17]). Therefore, under this assumption conical regularization scheme give in [17] holds, in particular $u_{\varepsilon}$ converge to $\bar{u}$. Another important property is that the regularized solutions $\left\{u_{\varepsilon}\right\}$ are uniformly bounded. Indeed, by the optimality and the fact $\bar{u}$ is feasible for regularized problems 1.2 , we get

$$
\left\|u_{\varepsilon}-u_{d}\right\|_{U}^{2} \leq J\left(u_{\varepsilon}\right) \leq J(\bar{u})
$$

and consequently,

$$
\left\|u_{\varepsilon}\right\|_{U} \leq\left\|u_{d}\right\|_{U}+\sqrt{J(\bar{u})}
$$

We begin with the following technical result.

Lemma 3.1. Let $\mu_{\varepsilon}^{*} \in K^{*}$ be a multiplier associated to 1.2 . Then, $\mu_{\varepsilon}^{*}(\bar{q}) \rightarrow 0$ as $\varepsilon \rightarrow 0$.

Proof. By the Taylor expansion of $J$ at $u=u_{\varepsilon}$, we have

$$
J(\bar{u})=J\left(u_{\varepsilon}\right)+D J\left(u_{\varepsilon}\right)\left(\bar{u}-u_{\varepsilon}\right)+\frac{1}{2} D^{2} J\left(u_{\theta}\right)\left(\bar{u}-u_{\varepsilon}, \bar{u}-u_{\varepsilon}\right),
$$


where $u_{\theta}=u_{\varepsilon}+\theta\left(\bar{u}-u_{\varepsilon}\right)$ with $\theta \in(0,1)$. By a direct computation of the derivatives, we get

$$
J(\bar{u})-J\left(u_{\varepsilon}\right)-D J\left(u_{\varepsilon}\right)\left(\bar{u}-u_{\varepsilon}\right)=\frac{\kappa}{2}\left\|\bar{u}-u_{\varepsilon}\right\|_{U}^{2}+\frac{1}{2}\left\|S \bar{u}-S u_{\varepsilon}\right\|_{V}^{2} .
$$

Applying KKT conditions 1.3a and $1.3 \mathrm{~b}$, we obtain

$$
D J\left(u_{\varepsilon}\right)\left(\bar{u}-u_{\varepsilon}\right)=-\left(\mu_{\varepsilon}^{*} \circ G\right)\left(\bar{u}-u_{\varepsilon}\right)=-\mu_{\varepsilon}^{*}\left(G \bar{u}-G u_{\varepsilon}\right)=-\mu_{\varepsilon}^{*}(G \bar{u}-w)=-\mu_{\varepsilon}^{*}(\bar{q}),
$$

and consequently

$$
\frac{\kappa}{2}\left\|\bar{u}-u_{\varepsilon}\right\|_{U}^{2}+\frac{1}{2}\left\|S \bar{u}-S u_{\varepsilon}\right\|_{V}^{2}=J(\bar{u})-J\left(u_{\varepsilon}\right)+\mu_{\varepsilon}^{*}(\bar{q}) .
$$

Equation (3.5) holds for every family of dilating cones $\left\{\mathcal{K}_{\varepsilon}\right\}$. Furthermore, since $u_{\varepsilon} \rightarrow \bar{u}$, the left-hand side term in 3.5 converges to 0 and we also have $J\left(u_{\varepsilon}\right) \rightarrow J(\bar{u})$, consequently, $\mu_{\varepsilon}^{*}(\bar{q}) \rightarrow 0$ as $\varepsilon \rightarrow 0$.

\subsection{Stability for the Henig conical regularization}

Let $\mu_{H, \varepsilon}^{*} \in Y^{*}$ be a multiplier associated with Henig regularized problem (3.1) for $\varepsilon$ small enough. Without loss of generality, we assume $\mu_{H, \varepsilon}^{*} \neq 0$. Otherwise, it follows from the KKT condition 1.3a that we have the trivial case $u_{\varepsilon}^{H}=\bar{u}$. Indeed, if $\mu_{H, \varepsilon}^{*}=0$, then by KKT condition (1.3a), we have

$$
D J\left(u_{\varepsilon}^{H}\right)=0 .
$$

Since $u_{\varepsilon}^{H} \rightarrow \bar{u}$ for $\varepsilon \rightarrow 0$, by the continuity of $D J$, we have

$$
D J(\bar{u})=0 .
$$

On the other hand, $\bar{u}$ is trivially a feasible point of problem (3.1), that is,

$$
G \bar{u}-w \leq \mathcal{K}_{\varepsilon} 0
$$

Conditions (3.6) and (3.7) imply that $\bar{u}$ verifies KKT conditions (1.3) with $\mu_{H, \varepsilon}^{*}=0$. Hence, $\bar{u}$ is a minimal point of problem (3.1). By the uniqueness of solution $u_{\varepsilon}^{H}=\bar{u}$.

Now, using the notation $\delta_{\varepsilon}^{H}:=\varepsilon\left\|\mu_{H, \varepsilon}^{*}\right\|_{Y^{*}}$, we have the following estimate.

Theorem 3.2. The sequence $\left\{\delta_{\varepsilon}^{H}\right\} \rightarrow 0$ as $\varepsilon \rightarrow 0$, and the following estimate holds:

$$
\frac{\kappa}{2}\left\|\bar{u}-u_{\varepsilon}^{H}\right\|_{U}^{2}+\frac{1}{2}\left\|S \bar{u}-S u_{\varepsilon}^{H}\right\|_{V}^{2} \leq J(\bar{u})-J\left(u_{\varepsilon}^{H}\right)-\delta_{\varepsilon}^{H}\left|\beta^{*}(\bar{q})\right| .
$$

Proof. By definition $\bar{q} \in-K$, and $\bar{q} \neq 0$ by assumption $(3.3)$. Therefore, $-\bar{q} / \beta^{*}(-\bar{q})$ is well defined and satisfies $-\bar{q} / \beta^{*}(-\bar{q}) \in \Theta$. By Theorem 2.1, we get

$$
\mu_{H, \varepsilon}^{*}\left(\frac{-\bar{q}}{\beta^{*}(-\bar{q})}\right)=\mu_{H, \varepsilon}^{*}\left(\frac{-\bar{q}}{\left|\beta^{*}(\bar{q})\right|}\right) \geq \varepsilon\left\|\mu_{H, \varepsilon}^{*}\right\|_{Y^{*}}=\delta_{\varepsilon}^{H} .
$$


By Lemma 3.1. we have $\mu_{H, \varepsilon}^{*}(\bar{q}) \rightarrow 0$, which due to the above inequality, implies that $\delta_{\varepsilon}^{H}=\varepsilon\left\|\mu_{H, \varepsilon}^{*}\right\|_{Y^{*}} \rightarrow 0$ as $\varepsilon \rightarrow 0$. Furthermore, we also have $\mu_{H, \varepsilon}^{*}(\bar{q}) \leq-\delta_{\varepsilon}^{H}\left|\beta^{*}(\bar{q})\right|$, which when combined with (3.5), yields (3.8).

We can establish an a priori estimate in term of the regularization parameter when problem (1.1) is regular, that is, when KKT conditions for problem (1.1) are solvable: There is a multiplier $\bar{\mu}^{*} \in K^{*}$ such that

$$
D J(\bar{u})+\bar{\mu}^{*} \circ G=0, \quad \bar{\mu}^{*}(G \bar{u}-w)=0, \quad G \bar{u}-w \leq_{K} 0 .
$$

Theorem 3.3. Assume that (1.1) is regular. Then, for $\varepsilon$ small enough there is a constant $c>0$, independent of $\varepsilon$, such that

$$
\frac{\kappa}{2}\left\|\bar{u}-u_{\varepsilon}^{H}\right\|_{U}^{2}+\frac{1}{2}\left\|S \bar{u}-S u_{\varepsilon}^{H}\right\|_{V}^{2} \leq c \varepsilon .
$$

Proof. By using the Taylor expansion (centered at $u=\bar{u}$ ), we have

$$
\begin{aligned}
J(\bar{u})-J\left(u_{\varepsilon}^{H}\right) & =J(\bar{u})-\left(J(\bar{u})+D J(\bar{u})\left(u_{\varepsilon}^{H}-\bar{u}\right)+\frac{\kappa}{2}\left\|\bar{u}-u_{\varepsilon}^{H}\right\|_{U}^{2}+\frac{1}{2}\left\|S \bar{u}-S u_{\varepsilon}^{H}\right\|_{V}^{2}\right) \\
& =-D J(\bar{u})\left(u_{\varepsilon}^{H}-\bar{u}\right)-\frac{\kappa}{2}\left\|\bar{u}-u_{\varepsilon}^{H}\right\|_{U}^{2}-\frac{1}{2}\left\|S \bar{u}-S u_{\varepsilon}^{H}\right\|_{V}^{2} \\
& \leq-D J(\bar{u})\left(u_{\varepsilon}^{H}-\bar{u}\right) .
\end{aligned}
$$

Since (1.1) is regular, by applying KKT conditions (3.9), we have

$$
J(\bar{u})-J\left(u_{\varepsilon}^{H}\right) \leq-D J(\bar{u})\left(u_{\varepsilon}^{H}-\bar{u}\right)=\left(\bar{\mu}^{*} \circ G\right)\left(u_{\varepsilon}^{H}-\bar{u}\right)=\bar{\mu}^{*}\left(G u_{\varepsilon}^{H}-w\right)=\bar{\mu}^{*}\left(q_{\varepsilon}^{H}\right) .
$$

On the other hand, since $q_{\varepsilon}^{H} \in-K_{\varepsilon}=-\operatorname{cl}\left[\operatorname{cone}\left(\Theta+\varepsilon B_{Y}\right)\right]$, we can take a sequence $\left\{q_{n, \varepsilon}\right\}_{n \in \mathbb{N}} \subset-\operatorname{cone}\left(\Theta+\varepsilon B_{Y}\right)$ such that

$$
q_{\varepsilon}^{H}=\lim _{n} q_{n, \varepsilon} .
$$

By definition, there exist $\alpha_{n, \varepsilon} \geq 0, \theta_{n, \varepsilon} \in \Theta, b_{n, \varepsilon} \in B_{Y}$ such that

$$
q_{n, \varepsilon}=-\alpha_{n, \varepsilon}\left(\theta_{n, \varepsilon}+\varepsilon b_{n, \varepsilon}\right)
$$

Applying linearity and positiviness of multiplier $\bar{\mu}^{*}$, we have

$$
\bar{\mu}^{*}\left(q_{n, \varepsilon}\right)=-\bar{\mu}^{*}\left(\alpha_{n, \varepsilon} \theta_{n, \varepsilon}\right)-\varepsilon \bar{\mu}^{*}\left(\alpha_{n, \varepsilon} b_{n, \varepsilon}\right) \leq \varepsilon \alpha_{n, \varepsilon}\left|\bar{\mu}^{*}\left(b_{n, \varepsilon}\right)\right| \leq \varepsilon \alpha_{n, \varepsilon}\left\|\bar{\mu}^{*}\right\|_{Y^{*}}
$$

Let us prove now that $\left\{\alpha_{n, \varepsilon}\right\}$ is uniformly bounded with respect to regularization parameter $\varepsilon$. By applying $\beta^{*}$ to expression (3.11), we have

$$
\beta^{*}\left(q_{n, \varepsilon}\right)=-\alpha_{n, \varepsilon}\left(\beta^{*}\left(\theta_{n, \varepsilon}\right)+\varepsilon \beta^{*}\left(b_{n, \varepsilon}\right)\right)=-\alpha_{n, \varepsilon}\left(1+\varepsilon \beta^{*}\left(b_{n, \varepsilon}\right)\right)
$$


and dividing this expression by $1+\varepsilon \beta^{*}\left(b_{n, \varepsilon}\right)$, we obtain

$$
\alpha_{n, \varepsilon}=-\frac{\beta^{*}\left(q_{n, \varepsilon}\right)}{1+\varepsilon \beta^{*}\left(b_{n, \varepsilon}\right)} \leq \frac{1}{1-\varepsilon\left\|\beta^{*}\right\|_{Y^{*}}}\left\|\beta^{*}\right\|_{Y^{*}}\left\|q_{n, \varepsilon}\right\|_{Y}=\frac{1}{1-\varepsilon}\left\|q_{n, \varepsilon}\right\|_{Y} \leq 2\left\|q_{n, \varepsilon}\right\|_{Y},
$$

where in the last step, we assumed, without loss of generality that $\varepsilon<1 / 2$. Consequently

$$
\alpha_{n, \varepsilon} \leq 2\left\|q_{n, \varepsilon}\right\|_{Y}
$$

and by substituting this expression in $(3.12)$, we have

$$
\bar{\mu}^{*}\left(q_{n, \varepsilon}\right) \leq \varepsilon 2\left\|q_{n, \varepsilon}\right\|_{Y}\left\|\bar{\mu}^{*}\right\|_{Y^{*}}
$$

Now taking limit, by 3.10 , we have

$$
\bar{\mu}^{*}\left(q_{\varepsilon}^{H}\right)=\lim _{n} \bar{\mu}^{*}\left(q_{n, \varepsilon}\right) \leq \varepsilon 2\left\|q_{\varepsilon}^{H}\right\|_{Y}\left\|\bar{\mu}^{*}\right\|_{Y^{*}}
$$

Since $q_{\varepsilon}^{H}=G u_{\varepsilon}^{H}-w$, by the continuity of $G$ and bound (3.4), we can take $\left\|q_{\varepsilon}^{H}\right\|_{Y} \leq c$, for some positive constant $c>0$, independent of $\varepsilon$. Using this in the previous inequality, we get

$$
\bar{\mu}^{*}\left(q_{\varepsilon}^{H}\right) \leq c \varepsilon .
$$

Consequently, we have

$$
J(\bar{u})-J\left(u_{\varepsilon}^{H}\right) \leq \bar{\mu}^{*}\left(q_{\varepsilon}^{H}\right) \leq c \varepsilon .
$$

Now substituting this expression in (3.8), we get

$$
\frac{\kappa}{2}\left\|\bar{u}-u_{\varepsilon}^{H}\right\|_{U}^{2}+\frac{1}{2}\left\|S \bar{u}-S u_{\varepsilon}^{H}\right\|_{V}^{2} \leq \varepsilon\left(c-\left\|\mu_{H, \varepsilon}^{*}\right\|_{Y^{*}}\left|\beta^{*}(\bar{q})\right|\right) .
$$

From (3.13), we can take another positive constant $c>0$, independent of $\varepsilon$, such that

$$
\frac{\kappa}{2}\left\|\bar{u}-u_{\varepsilon}^{H}\right\|_{U}^{2}+\frac{1}{2}\left\|S \bar{u}-S u_{\varepsilon}^{H}\right\|_{V}^{2} \leq c \varepsilon
$$

and $\left\|\mu_{H, \varepsilon}^{*}\right\|_{Y^{*}} \leq c$. The proof is complete.

In the above proof, we showed that for a regular problem, the set of regularized Lagrange multipliers $\left\{\left\|\mu_{H, \varepsilon}^{*}\right\|_{Y^{*}}\right\}$ is uniformly bounded for $\varepsilon$ sufficiently small. On the other hand, in [17, Theorem 3.4], assuming that $Y$ is a Hilbert constraint space, we proved that the norm boundedness of multipliers $\left\{\left\|\mu_{H, \varepsilon}^{*}\right\|_{Y^{*}}\right\}$ implies the the regularity of the original problem (1.1). Noting that the proof essentially used the reflexivity of the space $Y$ and the fact that from the norm-bounded multipliers, we can extract a weakly convergent subsequence whose weak limit is a Lagrange multiplier of the KKT system of (1.1), and hence proving that the problem (1.1) is regular. Following this idea, we can prove the following generalization relevant to the present setting. 
Proposition 3.4. Assume that $Y$ is reflexive. If the set of multipliers $\left\{\mu_{H, \varepsilon}^{*}\right\}$ is norm bounded for $\varepsilon$ small enough, then problem (1.1) is regular.

Therefore, from Theorem 3.3 and Proposition 3.4, we get the following characterization of regularity for problem 1.1 .

Corollary 3.5. Assume $Y$ is reflexive. Problem (1.1) is regular, if and only if, the set of multipliers $\left\{\mu_{H, \varepsilon}^{*}\right\}$ is norm bounded for $\varepsilon$ small enough.

\subsection{Stability for the constructible dilating regularization}

We now return to 3.2. We recall that $C_{\varepsilon}=\bigcap_{i \in I} H_{\lambda_{i, \varepsilon}^{*}}$, where $\lambda_{i, \varepsilon}^{*}$ are defined by 2.3. We also recall that $\left\{H_{\lambda_{i}^{*}}\right\}_{i \in I} \subset Y^{*}$ corresponds to a representation of $K$ where $\left\{\lambda_{i}^{*}\right\}_{i \in I}$ are of unit norm and $\beta^{*} \in K^{\sharp}$ is a strictly positive functional of unit norm. We continue to assume that the cone $K$ is given by a numerable representation. This condition is quite general and covers separable Banach spaces (see for example [2, Theorem 1]). Therefore, we will assume $I=\mathbb{N}$.

As before, for any multiplier associated with $(3.2)$, we take $\mu_{C, \varepsilon}^{*} \neq 0$, and define the quantity $\delta_{\varepsilon}^{C}=\varepsilon\left\|\mu_{C, \varepsilon}^{*}\right\|_{Y^{*}}$. By construction, $K_{\varepsilon} \subset C_{\varepsilon}$. Therefore $C_{\varepsilon}^{*} \subset K_{\varepsilon}^{*}$ and the dual of constructible dilating cone is contained on the dual of Henig dilating cones for each $\varepsilon \in(0,1)$. As a consequence, the characterization given in Theorem 2.1 also holds and we have

$$
\mu_{C, \varepsilon}^{*}(\theta) \geq \varepsilon\left\|\mu_{C, \varepsilon}^{*}\right\|_{Y^{*}} \quad \text { for every } \theta \in \Theta .
$$

Therefore the following result, which is an analogue of Theorem 3.2 holds.

Theorem 3.6. For $\varepsilon \rightarrow 0$, we have $\delta_{\varepsilon}^{C} \rightarrow 0$. Furthermore,

$$
\frac{\kappa}{2}\left\|\bar{u}-u_{\varepsilon}^{C}\right\|_{U}^{2}+\frac{1}{2}\left\|S \bar{u}-S u_{\varepsilon}^{C}\right\|_{V}^{2} \leq J(\bar{u})-J\left(u_{\varepsilon}^{C}\right)-\delta_{\varepsilon}^{C}\left|\beta^{*}(\bar{q})\right| .
$$

To obtain an analogue of Theorem 3.3, we require int $K \neq \emptyset$ and that the Slater constraint qualification holds: There exist $\bar{a} \in U$ such that

$$
G \bar{a}-w \in-\operatorname{int} K
$$

For any $y \in Y$, let us define the real sequence $\left\{\lambda_{i, \varepsilon}^{*}(y)\right\}_{i \in \mathbb{N}}$. By Lemma 2.2, we have

$$
\left|\lambda_{i, \varepsilon}^{*}(y)\right| \leq\left\|\lambda_{i, \varepsilon}^{*}\right\|_{Y^{*}}\|y\|_{Y} \leq\|y\|_{Y}
$$

and hence $\left\{\lambda_{i, \varepsilon}^{*}(y)\right\}_{i \in \mathbb{N}} \subset \ell^{\infty}$. We define the perturbed map $G_{\infty}: X \times \mathbb{R}_{+} \rightarrow \ell^{\infty}$ by

$$
G_{\infty}(x, \varepsilon):=\left\{\lambda_{i, \varepsilon}^{*}(G(x)-w)\right\}_{i \in \mathbb{N}}
$$


and for $\varepsilon \geq 0$ an associated family of the perturbed problems:

$$
J(u):=\frac{1}{2}\left\|S u-z_{d}\right\|_{V}^{2}+\frac{\kappa}{2}\left\|u-u_{d}\right\|_{U}^{2} \text { subject to } G_{\infty}(u, \varepsilon) \leq_{\ell_{+}^{\infty}} 0, u \in U .
$$

Due to the representation of a cone, $(3.15)$ and $(3.2)$ are equivalent. That is, they have the same feasible set and hence the same solution set. In the same way, problems (3.15) with $\varepsilon=0$ and $(1.1)$ are equivalent and their unique solutions coincide.

Theorem 3.7. For $\varepsilon \geq 0$, problem (3.15) has a unique solution $u_{\varepsilon}^{C}$.

For $q_{\bar{a}}:=G \bar{a}-w$, by (3.14 there exists $k>0$ such that $q_{\bar{a}}+k B_{Y} \subset-K$, and hence

$$
\lambda_{i}^{*}\left(q_{\bar{a}}\right) \leq-k \sup \left|\lambda_{i}^{*}\left(B_{Y}\right)\right|=-k\left\|\lambda_{i}^{*}\right\|_{Y^{*}}=-k \quad \text { for every } i \in I,
$$

which is equivalent to $\left\{\lambda_{i}^{*}\left(q_{\bar{a}}\right)\right\}_{i \in \mathbb{N}} \in-$ int $\ell_{+}^{\infty}$, and hence confirming the Slater constraint qualification

$$
\exists \bar{a} \in U \text { such that } G_{\infty}(\bar{a}, 0) \in-\operatorname{int} \ell_{+}^{\infty} .
$$

Theorem 3.8. If (3.14) holds, then for sufficiently small $\varepsilon$, there exists a positive constant $c$ which is independent of $\varepsilon$, and we have

$$
\left\|u_{\varepsilon}^{C}-\bar{u}\right\|_{U} \leq c \sqrt{\varepsilon}
$$

Proof. We apply [1, Theorem 2.5] by identifying $X \equiv U, Y \equiv \ell^{\infty}, K \equiv \ell_{+}^{\infty}, W \equiv \mathbb{R}_{+}$, $f \equiv J$, and $g \equiv-G_{\infty}$. Note that our setting is simpler than in [1] as the objective $J$ is parameter independent. Clearly (3.16) implies that $\bar{u}$ is a regular point (see 1 , Definition 2.1]). Moreover, the maps $J$ and $G_{\infty}$ are continuously differentiable and we have

$$
\begin{aligned}
D J(\bar{u})(u) & =\left\langle S^{*}\left(S \bar{u}-z_{d}\right), u\right\rangle_{V}+\kappa\left\langle\bar{u}-u_{d}, u\right\rangle_{U}, \\
D^{2} J(\bar{u})(u, u) & =\|S u\|_{V}^{2}+\kappa\|u\|_{U}^{2} .
\end{aligned}
$$

Therefore, conditions (A1) and (A3) in [1] hold automatically. Note that $J$ and $D J(\bar{u})$ are Lipschitz continuous on any neighborhood of $\bar{u}$. The map $G_{\infty}$ is also Lipschitz. Indeed, let $B_{\bar{u}}$ and $B_{0}$ be two neighborhoods of $\bar{u}$ and 0 , respectively. Then, for every $u_{1}, u_{2} \in B_{\bar{u}}$ and for every $\varepsilon_{1}, \varepsilon_{2} \in B_{0}$, we have

$$
\begin{aligned}
& \left\|G_{\infty}\left(u_{1}, \varepsilon_{1}\right)-G_{\infty}\left(u_{2}, \varepsilon_{2}\right)\right\|_{\ell_{\infty}}=\max _{i \in \mathbb{N}}\left|\lambda_{i, \varepsilon_{1}}^{*} \circ G\left(u_{1}\right)-\lambda_{i, \varepsilon_{2}}^{*} \circ G\left(u_{2}\right)\right| \\
= & \max _{i \in \mathbb{N}}\left|\left(\lambda_{i, \varepsilon_{1}}^{*}-\lambda_{i, \varepsilon_{2}}^{*}\right) G\left(u_{1}\right)+\lambda_{i, \varepsilon_{2}}^{*} G\left(u_{1}-u_{2}\right)\right| \\
= & \max _{i \in \mathbb{N}}\left|\left(\varepsilon_{1}-\varepsilon_{2}\right) \beta^{*}\left(G\left(u_{1}\right)\right)+\left(\varepsilon_{2}-\varepsilon_{1}\right) \lambda_{i}^{*}\left(G\left(u_{1}\right)\right)+\lambda_{i, \varepsilon_{2}}^{*} G\left(u_{1}-u_{2}\right)\right| \\
\leq & \left(\|G\|_{L(U, Y)} \max _{u \in B_{\bar{u}}}\|u\|_{U}+\|G\|\right)\left[\left|\varepsilon_{1}-\varepsilon_{2}\right|+\left\|u_{1}-u_{2}\right\|_{U}\right]
\end{aligned}
$$


by using $\left\|\lambda_{i}^{*}\right\|_{Y^{*}}=1,\left\|\beta^{*}\right\|_{Y^{*}}=1$ and $\left\|\lambda_{i, \varepsilon_{2}}^{*}\right\|_{Y^{*}} \leq 1$ (see Lemma 2.2. Hence, condition (A2) in [1] also holds. To verify (2.4) in [1], we use the Taylor expansion. For any feasible $u$ of (3.15), by the Taylor expansion of $J$ at $u=\bar{u}$, we have

$$
J(u)=J(\bar{u})+D J(\bar{u})(u-\bar{u})+\frac{1}{2} D^{2} J\left(u_{\theta}\right)(u-\bar{u}, u-\bar{u})
$$

where $u_{\theta}=\bar{u}+\theta(u-\bar{u}), \theta \in(0,1)$. Problem (3.15) is convex, therefore by minimality of $\bar{u}$ we have $D J(\bar{u})(u-\bar{u}) \geq 0$. Consequently, by applying (3.17), we get

$$
J(u)-J(\bar{u}) \geq \frac{1}{2} D^{2} J\left(u_{\theta}\right)(u-\bar{u}, u-\bar{u}) \geq \frac{\kappa}{2}\|u-\bar{u}\|_{U}^{2} .
$$

Therefore all the conditions of [1, Theorem 2.5] hold and as a result, there is a constant $c$, independent of $\varepsilon$, such that $\left\|u_{\varepsilon}^{C}-\bar{u}\right\|_{U} \leq c \sqrt{\varepsilon}$, for $\varepsilon$ sufficiently small.

As a consequence of Theorems 3.6 and 3.8 , by using the same arguments as in the Henig conical regularization, we can prove an analogous of Corollary 3.5. In this case, the following analogue of Proposition 3.4 holds.

Proposition 3.9. Assume $Y$ is reflexive. If the set of multipliers $\left\{\mu_{C, \varepsilon}^{*}\right\}$ is norm bounded for $\varepsilon$ small enough, then problem (1.1) is regular.

And the corresponding characterization of regularity for problem (1.1).

Corollary 3.10. Assume $Y$ is reflexive, and (3.14) holds. Problem (3.2) is regular, if and only if, the set of multipliers $\left\{\mu_{C, \varepsilon}^{*}\right\}$ is norm bounded.

\section{Application to $L^{p}$-constrained optimization problems}

Let $\Omega \subset \mathbb{R}^{n}$ be a bounded convex domain, and let $1 \leq p \leq \infty$. We set $Y=L^{p}(\Omega)$, the space of integrable functions on $\Omega$ and choose the cone $K$ to be the cone of its positive functions given by

$$
K=L_{+}^{p}(\Omega)=\left\{f \in L^{p}(\Omega): f(x) \geq 0 \text { a.e. in } \Omega\right\} .
$$

Denoting $y_{u}=G u$ for every $u \in U$, problem (1.1) takes the following form:

$$
\begin{gathered}
\text { Minimize } J(u):=\frac{1}{2}\left\|S u-z_{d}\right\|_{V}^{2}+\frac{\kappa}{2}\left\|u-u_{d}\right\|_{U}^{2} \\
\text { subject to } y_{u}(x) \leq w(x) \text { a.e. in } \Omega, u \in U .
\end{gathered}
$$

In the following by $\bar{u}_{p}$ we denote the unique solution of 4.1). We will now develop the constructible dilating regularization scheme studied in previous sections for problem 4.1. Note that 4.1 is an abstract optimization problem with $L^{p}(\Omega)$ constraints; such problems 
are ubiquitous in many models, particularly in PDE constrained optimization models (see for example $9,12,13,21)$. Another critical field where our results are applicable is of Mathematical Economics where a vast amount of real-world models lead to general $L^{p}$ constrained optimization problems, see for example [24]. In particular, we note [23] which offers a thoughtful discussion of $L^{p}$ constrained models in the economics context and is akin to the present research. In practical applications that lead to 4.1) for varying values of $p$, the central question is of the optimality of $p$, which is influenced by issues such as numerical efficiency, regularity, stability, existence, and optimality conditions. Here we present a detailed study of all the above-stated aspects for the constructible regularization.

The primary difficulty in such studies is the emptiness of the interior of the cone $C=L_{+}^{p}(\Omega)$ for $1 \leq p<\infty$ when $Y=L^{p}(\Omega)$. Hence the Slater constraint qualification fails to hold. For the case $p=\infty$, where the cone $C=L_{+}^{\infty}(\Omega)$ has a nonempty interior, and a Slater constraint qualification holds, the space $Y=L^{\infty}(\Omega)$ is neither reflexive nor separable. Fortunately, in both of these cases, we will show that the conical regularization offers a satisfactory framework. In the following, we will differentiate these two cases.

First we need to give a representation of the cone of positive functions $L_{+}^{p}(\Omega)$. For this, we consider a family of convex partitions $\left\{\Delta^{\delta}\right\}_{\delta>0}$ of $\Omega$, where $\delta$ is a real parameter, see 6, 14. Each partition $\left\{\Delta^{\delta}\right\}$ consists of a finite number of closed and convex sets $\left\{\Delta_{i}^{\delta}\right\} \subset \Omega(i=1, \ldots, T(\delta))$ such that $\sum_{\Delta_{i}^{\delta} \in \Delta^{\delta}}\left|\Delta_{i}^{\delta}\right|=|\Omega|$, where $T=T(\delta)=\# \Delta^{\delta}$ is the cardinality of $\left\{\Delta^{\delta}\right\}$, and $|\Omega|$ is the Lebesgue measure of $\Omega$. We assume that diameter of the family tends to zero, that is,

$$
\operatorname{diam}\left(\Delta^{\delta}\right)=\max _{i=1, \ldots, T(\delta)} \operatorname{diam}\left(\Delta_{i}^{\delta}\right) \rightarrow 0 \quad \text { as } \delta \rightarrow 0
$$

where without any loss of generality, in the sequel we set $\delta \equiv \operatorname{diam}\left(\Delta^{\delta}\right)$.

The family of half-spaces

$$
\left\{H_{\lambda_{i}^{\delta *}}\right\}_{i=1, \ldots, T(\delta) ; \delta>0}=\left\{f \in Y: \int_{\Delta_{i}^{\delta}} f(s) d s \geq 0 \text { for every } i=1, \ldots, T(\delta), \delta>0\right\}
$$

is a constructible ${ }^{1}$ representation of the cone of positive functions.

\subsection{Case $1 \leq p<\infty$}

For a given $p$, we denote by $q$ the conjugate exponent satisfying $q^{-1}+p^{-1}=1$. For $\delta>0$, we define $\lambda_{p, i}^{\delta *} \in L^{p}(\Omega)^{*}$ by

$$
\lambda_{p, i}^{\delta *}(f)=\left|\Delta_{i}^{\delta}\right|^{-(p-1) / p} \int_{\Delta_{i}^{\delta}} f(s) d s \quad \text { for every } f \in L^{p}(\Omega) .
$$

\footnotetext{
${ }^{1}$ We can always consider a numerable representation by taking a sequence $\delta_{n} \rightarrow 0^{+}$.
} 
By Hölder's inequality, we get $\left\|\lambda_{p, i}^{\delta *}\right\|_{L^{p}(\Omega)^{*}} \leq 1$, in fact $\left\|\lambda_{p, i}^{\delta *}\right\|_{L^{p}(\Omega)^{*}}=1$. The strictly functional $\beta_{p}^{*} \in K^{\natural}$ is defined by

$$
\beta_{p}^{*}(f)=|\Omega|^{-(p-1) / p} \int_{\Omega} f d s \quad \text { for every } f \in L^{p}(\Omega)
$$

Therefore, for each $\varepsilon, \delta>0$, the functionals $\lambda_{p, \varepsilon, i}^{\delta *}$ are given by

$$
\lambda_{p, \varepsilon, i}^{\delta *}(f)=(1-\varepsilon) \lambda_{p, i}^{\delta *}(f)+\varepsilon \beta_{p}^{*}(f)=(1-\varepsilon)\left|\Delta_{i}^{\delta}\right|^{-(p-1) / p} \int_{\Delta_{i}^{\delta}} f d s+\varepsilon|\Omega|^{-(p-1) / p} \int_{\Omega} f d s
$$

for every $f \in L^{p}(\Omega)$. The family $\left\{\lambda_{p, \varepsilon, i}^{\delta *}\right\}_{i=1, \ldots, T(\delta) ; \delta>0}$ is a representation of $C_{\varepsilon}$. Therefore, the regularized problems are defined as follows:

$$
\begin{aligned}
& \text { Minimize } J(u):=\frac{1}{2}\left\|S u-z_{d}\right\|_{V}^{2}+\frac{\kappa}{2}\left\|u-u_{d}\right\|_{U}^{2} \text { subject to } \\
& (1-\varepsilon)\left|\Delta_{i}^{\delta}\right|^{-(p-1) / p} \int_{\Delta_{i}^{\delta}}\left(y_{u}-w\right) d s+\varepsilon|\Omega|^{-(p-1) / p} \int_{\Omega}\left(y_{u}-w\right) d s \leq 0 \\
& \quad \text { for every } i=1, \ldots, T(\delta), \delta>0, u \in U .
\end{aligned}
$$

To derive an explicit KKT system, we need to give a characterization of the dual of $C_{\varepsilon}^{*}$. We will also give an explicit characterization of the Henig dilating cone $K_{\varepsilon}$ which is based on [17, Theorem 4.2]. Since $C_{\varepsilon}^{*} \subset K_{\varepsilon}^{*}$, we can apply this result to the elements of $C_{\varepsilon}^{*}$. Noting that the dual of $L^{p}(\Omega)^{*}$ can be isometrically identified with $L^{q}(\Omega)$, the following proof uses the same line of arguments as in [17, Theorem 4.2]. In the following, for any $\mu^{*} \in L^{p}(\Omega)^{*}$, the associated Riesz element is denoted by $\mu \in L^{q}(\Omega)$. That is, $\mu \in L^{q}(\Omega)$ such that

$$
\mu^{*}(f)=\int_{\Omega} f(s) \mu(s) d s \quad \text { for every } f \in L^{p}(\Omega) .
$$

Proposition 4.1. Assume $1 \leq p<\infty, Y=L^{p}(\Omega), C=L_{+}^{p}(\Omega)$, and let $K_{\varepsilon}$ be the associated Henig dilating cone. The dual of this cone is given by

$$
K_{\varepsilon}^{*}=\left\{\mu^{*} \in L_{+}^{p}(\Omega)^{*}: \mu(x) \geq \varepsilon|\Omega|^{-(p-1) / p}\|\mu\|_{L^{q}(\Omega)} \text { a.e. in } \Omega\right\} .
$$

Proof. For $Y=L^{2}(\Omega)$, in 17 , Theorem 4.2], it was proven that

$$
K_{\varepsilon}^{*}=\left\{\mu^{*} \in L_{+}^{2}(\Omega)^{*}: \mu(x) \geq \varepsilon|\Omega|^{-1 / 2}\|\mu\|_{L^{2}(\Omega)} \text { a.e. in } \Omega\right\} .
$$

This result was based on [17, Theorem 4.1], and in this case we take into account that the base is defined by a normalized strictly functional. We have seen that Theorem 2.1 is an analogue of this result for a general Banach space. Therefore applying Theorem 2.1 and following the same steps as in the proof of [17, Theorem 4.2], we can easily extend this result to $Y=L^{p}(\Omega)$ for every $p \in[1, \infty)$. 
We have the following general result.

Theorem 4.2. For each $\varepsilon>0$, there exists unique solution $u_{p, \varepsilon}^{C}$ of problem (4.2). The solution sequence $u_{p, \varepsilon}^{C}$ converges to $\bar{u}_{p}$ as $\varepsilon \rightarrow 0$. Furthermore, for each $\varepsilon>0$, there exists a Lagrange multiplier $\mu_{C, p, \varepsilon}^{*} \in L_{+}^{p}(\Omega)^{*}$ such that

$$
\begin{gathered}
D J\left(u_{p, \varepsilon}^{C}\right)+\mu_{C, p, \varepsilon}^{*} \circ G=0 \text { in } U^{*}, \\
\int_{\Omega} \mu_{C, p, \varepsilon}\left(y_{u_{p, \varepsilon}^{C}}-w\right) d s=0, \\
\mu_{C, p, \varepsilon}(s) \geq \varepsilon|\Omega|^{-(p-1) / p}\left\|\mu_{C, p, \varepsilon}\right\|_{L^{q}(\Omega)} \text { a.e. in } \Omega, \\
(1-\varepsilon)\left|\Delta_{i}^{\delta}\right|^{-(p-1) / p} \int_{\Delta_{i}^{\delta}}\left(y_{u_{p, \varepsilon}^{C}}-w\right) d s+\varepsilon|\Omega|^{-(p-1) / p} \int_{\Omega}\left(y_{u_{p, \varepsilon}^{C}}-w\right) d s \leq 0
\end{gathered}
$$

for every $i=1, \ldots, T(\delta), \delta>0$. Finally, for sufficiently small $\varepsilon$, there exists a constant $c>0$, independent of $\varepsilon$, such that

$$
\frac{\kappa}{2}\left\|\bar{u}_{p}-u_{p, \varepsilon}^{C}\right\|_{U}^{2} \leq J\left(\bar{u}_{p}\right)-J\left(u_{p, \varepsilon}^{C}\right)-c \varepsilon\left\|\mu_{C, p, \varepsilon}\right\|_{L^{q}(\Omega)} .
$$

Proof. The existence is a standard result. The convergence and the optimality follows by applying conical regularization scheme, where we also use the dual characterization given in Proposition 4.1. The final statement is a direct consequence of Theorem 3.6.

\subsection{Case $p=\infty$}

We now take $Y=L^{\infty}(\Omega)$ and $K=L_{+}^{\infty}(\Omega)$. As noticed before, the cone $K$ now has a nonempty interior, however, we have lost the reflexivity and the separability of $Y$. For this case, the unit normal representation is defined by

$$
\lambda_{\infty, i}^{\delta *}(f)=\left|\Delta_{i}^{\delta}\right|^{-1} \int_{\Delta_{i}^{\delta}} f(s) d s,
$$

and the corresponding regularized problems read:

$$
\begin{aligned}
& \text { Minimize } J(u):=\frac{1}{2}\left\|S u-z_{d}\right\|_{V}^{2}+\frac{\kappa}{2}\left\|u-u_{d}\right\|_{U}^{2} \text { subject to } \\
& (1-\varepsilon)\left|\Delta_{i}^{\delta}\right|^{-1} \int_{\Delta_{i}^{\delta}}\left(y_{u}-w\right) d s+\varepsilon|\Omega|^{-1} \int_{\Omega}\left(y_{u}-w\right) d s \leq 0 \\
& \text { for every } i=1, \ldots, T(\delta), \delta>0, u \in U
\end{aligned}
$$

The Slater condition now reads: There is a constant $k>0$ and an element $\bar{a} \in U$ such that

$$
y_{\bar{a}}(x)-w(x) \leq-k<0 \quad \text { a.e. in } \Omega .
$$


Under this condition, the problem is regular and the KKT conditions remain valid. However, the need of regularization arises to circumvent the difficulties in the discretization due to the fact that the dual $L_{+}^{\infty}(\Omega)^{*}$ is non-separable. As it turns out, the regularized problems (4.4) provide a natural discretization scheme by fixing the regularization parameter and the diameter of the partition. For this, we apply an a priori estimate given in Theorem 3.8 .

Theorem 4.3. Assume that 4.5 holds. Then for each $\varepsilon>0$, there is unique solution $u_{\infty, \varepsilon}^{C}$ to problem (4.4). Furthermore, for sufficiently small $\varepsilon$, there exists a constant $c>0$, independent of $\varepsilon$, such that

$$
\left\|u_{\infty, 0}-u_{\infty, \varepsilon}^{C}\right\|_{U} \leq c \sqrt{\varepsilon}
$$

\section{A numerical experiment}

To verify the practical applicability of the proposed scheme, we conduct a numerical experiment for a known optimization problem (see [3, Example 3.20]) with non-regular $L^{p}$ multiplier for $1 \leq p<\infty$ but regular for $p=\infty$.

For $1 \leq p \leq \infty$, we set $U=V=\mathbb{R}, \Omega=[0,1], Y=L^{p}[0,1]$, and $K=L_{+}^{p}[0,1]$ the cone of positive functions. Let $G: \mathbb{R} \rightarrow L^{p}[0,1]$ be a linear bounded map defined by $y_{u}=-u_{F}$ for every $u \in \mathbb{R}$, where $u_{F}$ denotes the constant map given by $u_{F}(t)=u$ for every $t \in[0,1]$. Similarly, we define $w \in L^{p}[0,1]$ by $w(t)=-t$ for every $t \in[0,1]$.

Given the above data set, we consider the following optimization problem

$$
\text { Minimize } J(u)=\frac{1}{2} u^{2} \text { subject to } y_{u}(t) \leq w(t) \text { a.e. in }[0,1], u \in \mathbb{R} \text {. }
$$

Evidently, problem is of kind (5.1) by setting $S=z_{d}=u_{d}=0$, and $\kappa=1$. The unique solution of (5.1) for every $1 \leq p \leq \infty$ are $\bar{u}=\bar{u}_{p}=1$. We will consider the strictly functional(s)

$$
\beta^{*}(f)=\beta_{p}^{*}(f)=\int_{0}^{1} f(s) d s \quad \text { for every } f \in L_{+}^{p}[0,1] .
$$

For each $n \in \mathbb{N}$, we consider the convex partitions $\Delta^{\delta_{n}}$ of $\Omega=[0,1]$, with diameter $\delta_{n}=n^{-1}$, given by subintervals $\Delta_{i}^{\delta_{n}}=[(i-1) / n, i / n]$ for every $i=1, \ldots, n$. The family of partitions $\left\{\Delta^{\delta_{n}}\right\}_{n>0}$ verify the condition $\left\{\delta_{n}\right\} \rightarrow 0$ for $n \rightarrow \infty$. For the case $p<\infty$, the corresponding family of constructible regularized problems is given by

$$
\begin{gathered}
\text { Minimize } J(u)=\frac{1}{2} u^{2} \text { subject to } \\
(1-\varepsilon) n^{(p-1) / p} \int_{(i-1) / n}^{i / n}\left(-u_{F}+s\right) d s+\varepsilon \int_{0}^{1}\left(-u_{F}+s\right) d s \leq 0
\end{gathered}
$$

for every $i=1, \ldots, n, n \in \mathbb{N}, u \in \mathbb{R}$. 
For the case $p=\infty$, corresponding family of constructible regularized problems is given by

$$
\begin{aligned}
& \text { Minimize } J(u)=\frac{1}{2} u^{2} \text { subject to } \\
& (1-\varepsilon) n \int_{(i-1) / n}^{i / n}\left(-u_{F}+s\right) d s+\varepsilon \int_{0}^{1}\left(-u_{F}+s\right) d s \leq 0
\end{aligned}
$$

for every $i=1, \ldots, n, n \in \mathbb{N}, u \in \mathbb{R}$.

Condition (4.5) is verified, for example by taking $\bar{a}=2$. This is a Slater condition for the case $p=\infty$, so (5.3) admit KKT conditions. In general, if $p<\infty$, problem (5.1) is not regular since KKT conditions are unsolvable (see [3, Example 3.20]).

For $p<\infty$, by a direct computation, for every $i=1, \ldots, n, n \in \mathbb{N}, u \in \mathbb{R}$, we have

$$
\begin{aligned}
\lambda_{p, \varepsilon, i}^{\delta *}\left(y_{u}-w\right) & =(1-\varepsilon) n^{(p-1) / p} \int_{(i-1) / n}^{i / n}(-u+s) d s+\varepsilon \int_{0}^{1}(-u+s) d s \\
& =\frac{1}{2 n^{2}} n^{(p-1) / p}(1-\varepsilon)(-2 n u+2 i-1)+\varepsilon\left(-u+\frac{1}{2}\right)
\end{aligned}
$$

and, in particular

$$
\lambda_{p, \varepsilon, i}^{\delta *}\left(y_{u}-w\right) \leq 0 \quad \Longleftrightarrow \quad u \geq \frac{n^{(p-1) / p}(1-\varepsilon)(2 i-1)+\varepsilon n^{2}}{n^{(p-1) / p}(1-\varepsilon) 2 n+2 \varepsilon n^{2}}
$$

Therefore,

$$
u \geq \frac{1}{2} \frac{n^{(p-1) / p}(1-\varepsilon)(2 n-1)+\varepsilon n^{2}}{n^{(p-1) / p}(1-\varepsilon) n+\varepsilon n^{2}}
$$

for every $n \in \mathbb{N}, u \in \mathbb{R}$. Since we are minimizing $\frac{1}{2} u^{2}$, the solution to 5.2 is the maximum of the right-hand side of previous inequality, that is,

$$
u_{p, \varepsilon}^{C}=\max _{n \in \mathbb{N}} \beta_{p, \varepsilon}(n),
$$

where $\beta_{p, \varepsilon}(n):=\frac{1}{2} \frac{n^{(p-1) / p}(1-\varepsilon)(2 n-1)+\varepsilon n^{2}}{n^{(p-1) / p}(1-\varepsilon) n+\varepsilon n^{2}}$.

For the case $p=\infty$, we can compute explicitly the solution. Indeed

$$
u \geq \frac{1}{2} \frac{n(1-\varepsilon)(2 n-1)+\varepsilon n^{2}}{n(1-\varepsilon) n+\varepsilon n^{2}}=1-\frac{\varepsilon}{2}+\frac{\varepsilon-1}{2 n}
$$

for every $n \in \mathbb{N}$. From this, it follows easily that

$$
u_{\infty, \varepsilon}^{C}=\max _{n \in \mathbb{N}}\left(1-\frac{\varepsilon}{2}+\frac{\varepsilon-1}{2 n}\right)=1-\frac{\varepsilon}{2} .
$$

Therefore

$$
\left|u_{\infty, \varepsilon}^{C}-\bar{u}\right|=\frac{\varepsilon}{2}
$$


verifying 4.6. In fact, bound (4.6) is conservative, since

$$
E_{\infty}^{C}(\varepsilon):=\left|u_{\infty, \varepsilon}^{C}-\bar{u}\right|=\mathcal{O}(\varepsilon)
$$

In general, we will denote the error functional

$$
E_{p}^{C}(\varepsilon):=\left|u_{p, \varepsilon}^{C}-\bar{u}\right|
$$

For the case $p<\infty$, we approximate numerically these quantities by using Geogebra 11. To check numerically the convergence rates, we consider the Experimental Order of Convergence (EOC). Given a finite list of decreasing steps $\left\{\varepsilon_{1}, \ldots, \varepsilon_{N}\right\}$, we define the EOC at level $i$ for error functional $E_{p}^{C}$ by the following expression

$$
\operatorname{EOC}\left(E_{p}^{C}\right)=\frac{\ln E_{p}^{C}\left(\varepsilon_{i}\right)-\ln E_{p}^{C}\left(\varepsilon_{i-1}\right)}{\ln \varepsilon_{i}-\ln \varepsilon_{i-1}} \text { for every } i=2, \ldots, N
$$

In our computation we take $\varepsilon_{i}=1 \mathrm{e}-01 \varepsilon_{i-1}$. In numerical experiments, $\operatorname{EOC}\left(E_{p}^{C}\right)$ remained constant for small $\varepsilon_{i}$, providing a meaningful approximation of the real order of convergence $\mathcal{O}\left(E_{p}^{C}\right)$. In Table 5.1 we show these values for several values of $p$, while in Figure 5.1 we plot the Experimental Order of Convergence for $p \in(1,500)$. These results confirm

$$
\lim _{p \rightarrow \infty} \mathcal{O}\left(E_{p}^{C}\right)=1=\mathcal{O}\left(E_{\infty}^{C}\right)
$$

as predicted by (5.4). From this we note that is possible to find an $L^{p}$ approximation, for a small value of $p$, such that the accuracy is similar to the one obtained for $L^{\infty}$.

\begin{tabular}{|c|c|c|c|}
\hline$p$ & $\operatorname{EOC}\left(E_{p}^{C}\right)$ & $p$ & $\mathrm{EOC}\left(E_{p}^{C}\right)$ \\
\hline 1 & 0.5 & 20 & 0.952 \\
\hline 2 & 0.667 & 40 & 0.976 \\
\hline 3 & 0.75 & 50 & 0.98 \\
\hline 4 & 0.8 & 60 & 0.984 \\
\hline 5 & 0.833 & 80 & 0.988 \\
\hline 6 & 0.857 & 100 & 0.990 \\
\hline 7 & 0.875 & 200 & 0.995 \\
\hline 8 & 0.889 & 300 & 0.997 \\
\hline 9 & 0.9 & 400 & 0.998 \\
\hline 10 & 0.909 & 500 & 0.998 \\
\hline
\end{tabular}

Table 5.1: Experimental Order of Convergence for Constructible Regularization. 


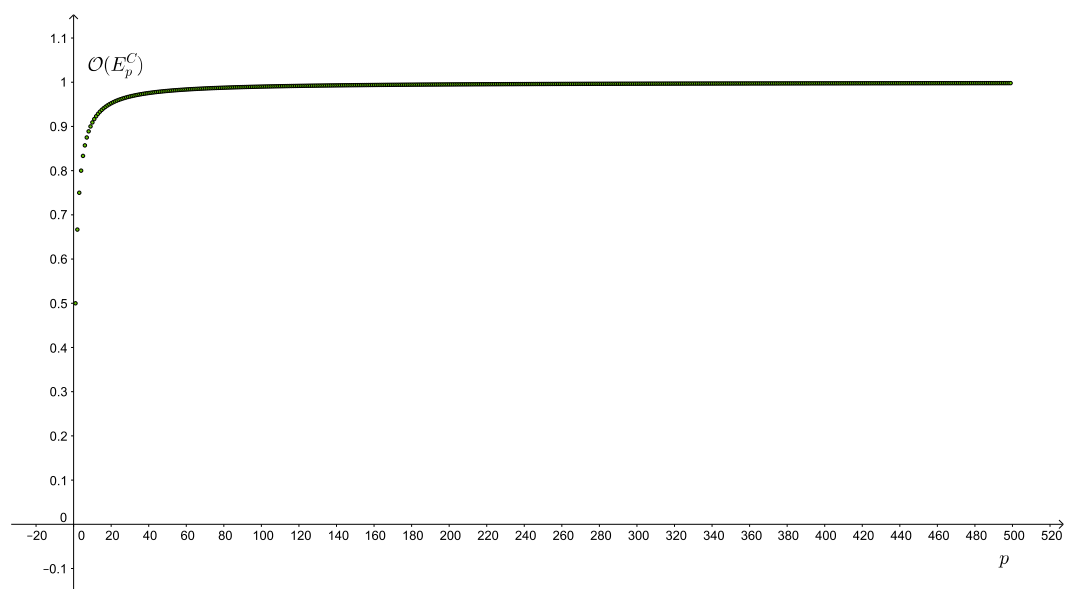

Figure 5.1: $\operatorname{EOC}\left(E_{p}^{C}\right)$ for $p \in(1,500)$.

In the second part of the experiment, restricting only to the case $p<\infty$, we compare the constructible dilating cone regularization and the Henig regularization. For a fixed $p \in[1, \infty)$, let $\left\{K_{\varepsilon}\right\}$ be the Henig dilating cones of $L_{+}^{p}[0,1]$ associated with the basis

$$
\Theta=\left\{f \in L_{+}^{p}[0,1]: \int_{0}^{1} f(s) d s=1\right\} .
$$

We consider the regularized problems

$$
\text { Minimize } J(u)=\frac{1}{2} u^{2} \text { subject to } y_{u}(t) \leq_{K_{\varepsilon}} w(t) \text { a.e. in }[0,1], u \in \mathbb{R} \text {. }
$$

Due to the lack of an analytic solution, we rely on a numerical approximation by using the Lagrange dual problem which can be computed explicitly. The Lagrangian reads

$$
L\left(u, \mu^{*}\right)=\frac{1}{2} u^{2}+\mu^{*} \circ(G u-w)=\frac{1}{2} u^{2}-u \int_{0}^{1} \mu d x+\int_{0}^{1} \mu x d x \quad \text { for every } \mu^{*} \in K_{\varepsilon}^{*},
$$

and the associated Lagrangian dual function is given by

$$
\begin{aligned}
g\left(\mu^{*}\right) & =\max _{u \in \mathbb{R}} L\left(u, \mu^{*}\right)=L\left(\int_{0}^{1} \mu d x, \mu^{*}\right)=\frac{1}{2} u^{2}-u \int_{0}^{1} \mu d x+\int_{0}^{1} \mu x d x \\
& =\int_{0}^{1} \mu x d x-\frac{1}{2}\left(\int_{0}^{1} \mu d x\right)^{2}
\end{aligned}
$$

for every $\mu^{*} \in K_{\varepsilon}^{*}$. By applying the characterization of $K_{\varepsilon}^{*}$ established in (4.3), the dual Lagrange problem is given by

$$
\begin{aligned}
& \text { Maximize } g(\mu)=\int_{0}^{1} \mu x d x-\frac{1}{2}\left(\int_{0}^{1} \mu d x\right)^{2} \\
& \text { subject to } \mu(x) \geq \varepsilon\|\mu\|_{L^{q}[0,1]} \text { a.e. in }[0,1] .
\end{aligned}
$$


In this case strong duality holds, 5.6 is uniquely solvable, and the solution $\mu_{H, p, \varepsilon}^{*}$ is a regular multiplier of problem (5.5). Furthermore, optimal values of (5.5) and (5.6) coincide, and we can recover solution $u_{p, \varepsilon}^{H}$ by formula

$$
u_{p, \varepsilon}^{H}=\int_{0}^{1} \mu_{H, p, \varepsilon} d x .
$$

To compute numerically the solution to (5.6), we consider a fixed partition $\left\{\Delta^{\delta_{n}}\right\}$ of $n=1000$ points. Each integral is approximated by using a Simpson's rule. In this case, we have solved numerically these problems by using CVX (see [8]).

As in constructible regularization case, the regularization error decrease for bigger $p$, and the blow up rate $\left\{\left\|\mu_{H, p, \varepsilon}\right\|_{L^{q}(\Omega)}\right\}$ is slower. In this case, we must also consider the influence of the discretization error. We have experienced that for the larger values of $p$, the regularization error soon stagnates below some threshold as the discretization error start dominating. This is a known effect in this kind of regularization methods, for example in optimal control. Below this threshold, see Figure 5.2, one can observe that the convergence rate of the regularization error and the blow-up rate of the multiplier are nearly of the same order for the two regularization methods.
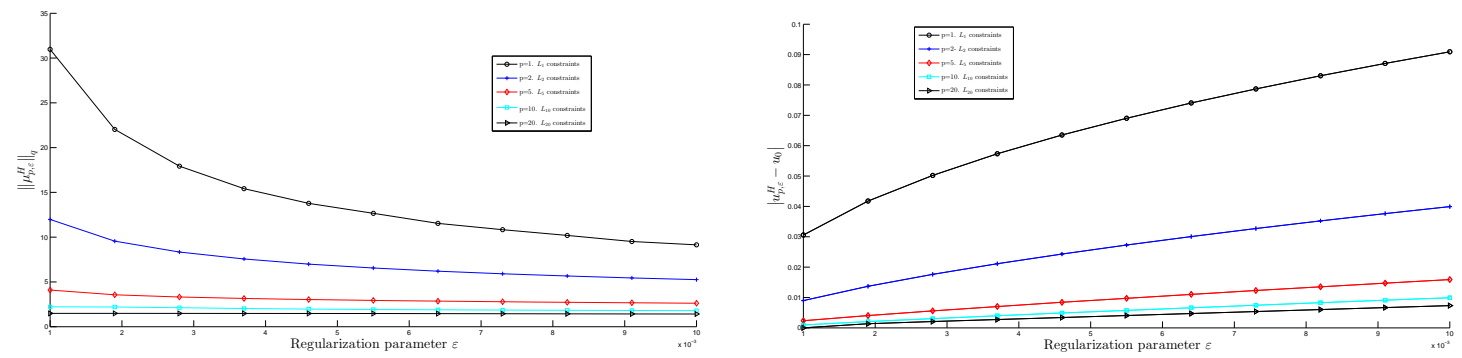

Figure 5.2: The first figure compare the blow-up rates for the Henig regularized multipliers and the second figure compares the convergent rates for the Henig regularized solutions.

\section{Acknowledgments}

Jadamba and Khan are supported by National Science Foundation Grant Number 1720067. Sama's work is partially supported by Ministerio de Economíay Competitividad (Spain), project MTM2015-68103-P and 2017-MAT12 (ETSI Industriales, UNED).

\section{References}

[1] W. Alt, Stability of solutions for a class of nonlinear cone constrained optimization problems I: Basic theory, Numer. Funct. Anal. Optim. 10 (1989), no. 11-12, 10531064. 
[2] D. Azagra and J. Ferrera, Every closed convex set is the set of minimizers of some $C^{\infty}$-smooth convex function, Proc. Amer. Math. Soc. 130 (2002), no. 12, 3687-3692.

[3] J. F. Bonnans and A. Shapiro, Perturbation Analysis of Optimization Problems, Springer Series in Operations Research, Springer-Verlag, New York, 2000.

[4] J. M. Borwein and J. D. Vanderwerff, Constructible convex sets, Set-Valued Anal. 12 (2004), no. 1-2, 61-77.

[5] J. M. Borwein and D. Zhuang, Super efficiency in vector optimization, Trans. Amer. Math. Soc. 338 (1993), no. 1, 105-122.

[6] O. Davydov, Approximation by piecewise constants on convex partitions, J. Approx. Theory 164 (2012), no. 2, 346-352.

[7] A. Göpfert, H. Riahi, C. Tammer and C. Zălinescu, Variational Methods in Partially Ordered Spaces, CMS Books in Mathematics/Ouvrages de Mathématiques de la SMC 17, Springer-Verlag, New York, 2003.

[8] M. Grant and S. Boyd, CVX: Matlab Software for Disciplined Convex Programming, Version 2.1. http://cvxr.com/cvx

[9] M. D. Gunzburger, Perspectives in Flow Control and Optimization, Advances in Design and Control 5, Society for Industrial and Applied Mathematics (SIAM), Philadelphia, PA, 2003.

[10] M. I. Henig, Proper efficiency with respect to cones, J. Optim. Theory Appl. 36 (1982), no. 3, 387-407.

[11] M. Hohenwarter, GeoGebra: Ein Softwaresystem für dynamische Geometrie und Algebra der Ebene, Paris Lodron University, Salzburg, Austria, 2002.

[12] V. Isakov, Inverse Problems for Partial Differential Equations, Second edition, Applied Mathematical Sciences 127, Springer, New York, 2006.

[13] K. Ito and K. Kunisch, Lagrange Multiplier Approach to Variational Problems and Applications, Advances in Design and Control 15, Society of Industrial and Applied Mathematics (SIAM), Philadelphia, PA, 2008.

[14] B. Jadamba, A. A. Khan and M. Sama, Regularization for state constrained optimal control problems by half spaces based decoupling, Systems Control Lett. 61 (2012), no. 6, 707-713. 
[15] _ Error estimates for integral constraint regularization of state-constrained elliptic control problems, Comput. Optim. Appl. 67 (2017), no. 1, 39-71.

[16] B. Jiménez, V. Novo and M. Sama, An extension of the basic constraint qualification to nonconvex vector optimization problems, J. Global Optim. 56 (2013), no. 4, 17551771.

[17] A. A. Khan and M. Sama, A new conical regularization for some optimization and optimal control problems: convergence analysis and finite element discretization, $\mathrm{Nu}-$ mer. Funct. Anal. Optim. 34 (2013), no. 8, 861-895.

[18] A. A. Khan, C. Tammer and C. Zălinescu, Set-valued Optimization: An Introduction with Applications, Vector Optimization, Springer, Heidelberg, 2015.

[19] P. Kogut, G. Leugering and R. Schiel, On Henig regularization of material design problems for quasi-linear p-biharmonic equation, Appl. Math. 7 (2016), no. 14, 1547 1570.

[20] - On the relaxation of state-constrained linear control problems via Henig dilating cones, Control Cybernet. 45 (2016), no. 2, 131-162.

[21] I. Lasiecka and R. Triggiani, Control Theory for Partial Differential Equations: Continuous and Approximation Theories I: Abstract Parabolic Systems, Encyclopedia of Mathematics and its Applications 74, Cambridge University Press, Cambridge, 2000.

[22] E. K. Makarov and N. N. Rachkovski, Unified representation of proper efficiency by means of dilating cones, J. Optim. Theory Appl. 101 (1999), no. 1, 141-165.

[23] K. Martin, C. T. Ryan and M. Stern, The Slater conundrum: duality and pricing in infinite-dimensional optimization, SIAM J. Optim. 26 (2016), no. 1, 111-138.

[24] A. Mas-Colell and W. R. Zame, Equilibrium theory in infinite-dimensional spaces, in: Handbook of Mathematical Economics IV, 1835-1898, Handbooks in Econom. 1, North-Holland, Amsterdam, 1991.

[25] R. E. Megginson, An Introduction to Banach Space Theory, Graduate Texts in Mathematics 183, Springer-Verlag, New York, 1998.

Baasansuren Jadamba and Akhtar A. Khan

Center for Applied and Computational Mathematics, School of Mathematical Sciences, Rochester Institute of Technology, 85 Lomb Memorial Drive, Rochester, New York, 14623, USA

E-mail address: bxjsma@rit.edu, aaksma@rit.edu 


\section{Miguel Sama}

Departamento de Matemática Aplicada, Universidad Nacional de Educación a Distancia, Calle Juan del Rosal, 12, 28040 Madrid, Spain

E-mail address: msama@ind.uned.es 